\title{
A case of orbital granulocytic sarcoma (chloroma) well responded to the treatment
}

\begin{abstract}
Granulocytic sarcoma (GS), myeloid sarcoma (MS) or extra medullary leukemic deposits is an unusual manifestation of AML, accounting for about 3\% of acute leukaemia's. GS may occur as a manifestation of a systemic myelogenic leukaemia or it may precede systemic manifestations of peripheral blood and bone marrow. When GS precedes the development of systemic leukemia the diagnosis can be difficult for clinicians. Owing to the rarity of GS there are no large studies analyzing prognostic factors in these patients. The presence of MS at diagnosis in a leukaemic patient was traditionally considered to represent a marker for poor clinical outcome and shorter survival. Herein, we described a patient with newly diagnosed AML and right orbital GS which treated with standard chemotherapy regime and responded dramatically in two weeks. An extra-medullary leukemic infiltration is a bad prognostic sign, because it might act as a reservoir for proliferation of leukaemic cells and an eventual relapse. In addition to good medical response of our case we want to note that a variety of ophthalmologic findings can be seen in leukaemia's. Careful and detailed ophthalmologic examination plays crucial role in timely diagnosis of many curable disordered including leukaemia's and has vital importance on the management of leukaemia's.
\end{abstract}

Abbreviations: GS, granulocytic sarcoma; MS, myeloid sarcoma; AML, acute myeloid leukemia; MDS, myelodysplastic syndrome; MPS, myeloproliferative disorders

\section{Introduction}

Acute myeloid leukemia (AML) accounts for nearly $15 \%$ of all leukemia's in children. ${ }^{1,2}$ The leukemic cells can infiltrate any extramedullary site, tumorous accumulations within soft tissues and bones being labeled as granulocytic sarcomas. Granulocytic sarcoma (GS) or extramedullary leukemic deposits is an unusual manifestation of AML, accounting for about $3 \%$ of cases of AML. GS was firstly defined in 1811 by Allen Burns who described a green tumour involving the orbit. Because these tumours can exhibit a characteristic green colour they were named chloroma. The green colour is caused by exposure of the enzyme myeloperoxidase to ultraviolet light. However, the gross appearance of these tumours is variable and up to $30 \%$ of granulocytic sarcomas do not display a green colour. This led Rappaport to rename these tumours granulocytic sarcoma in $1966 .{ }^{1-5}$

GS may occur as a manifestation of a systemic myelogenic leukaemia or it may precede systemic manifestations of peripheral blood and bone marrow. When the chloroma precedes the development of systemic leukemia the diagnosis can be difficult for clinicians. In addidion to acute myeloid leukemias, it also can be seen in chronic myeloid leukemia, myelodisplastic syndromes, hypereosinophilic syndromes, and polycytemias. The diferential diagnosis of orbital tumors includes malignant lymphoma, rhabdomyosarcoma, and neuroblastoma. Owing to the rarity of GS there are no large studies analyzing prognostic factors in these patients. Few reports compare the prognosis of isolated GS with patients with either GS with concomitant AML or AML presenting without MS, making the contributing effect of MS on prognosis difficult to assess. Overall, however, it appears that there is a difference in prognosis between patients presenting with isolated MS compared with MS patients with either concomitant AML or at AML relapse. The presence of MS at diagnosis in a leukemic patient was traditionally considered to represent a marker for poor clinical outcome and shorter survival. ${ }^{2-6}$ Herein, we described a patient with newly diagnosed AML and right orbital GS which treated with standard chemotherapy regime and responded dramatically in two weeks.

\section{Case report}

Eight years-old girl admitted to our ophthalmology clinic with confusion, lethargy, pale color and right orbital swelling growing in last 2 weeks. Anterior and posterior segments were evaluated at ophthalmologic examination. At orbital examination, Hertel exophthalmometry and unenhanced and contrast-enhanced orbital MRI results were evaluated. At ophthalmologic examination, visual acuity was $10 / 10$, intraocular pressure was $16 \mathrm{mmHg}$ for both eyes. There was proptosis at right eye. Hertel exophthalmometry at $110 \mathrm{~mm}$ was $20 \mathrm{~mm}$ for right and $16 \mathrm{~mm}$ for left eye. At fundus examination, minimal retinal hemorrhages were present at right eye. Palpation of the right eyelid showed approximately $20 \times 15 \mathrm{~mm}$ mass which is stiff, immobile and fixed to bone and located at upper medial orbital region (Figures 1a-2b).

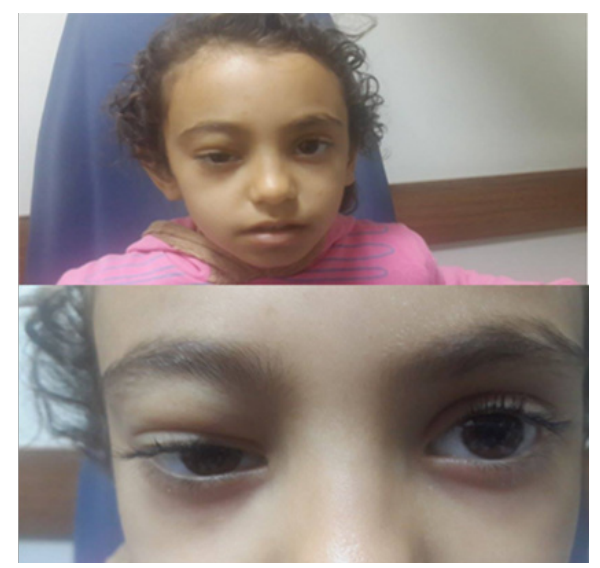

Figure Ia \& Ib Proptosis in the right eye of the patient due to GS. 


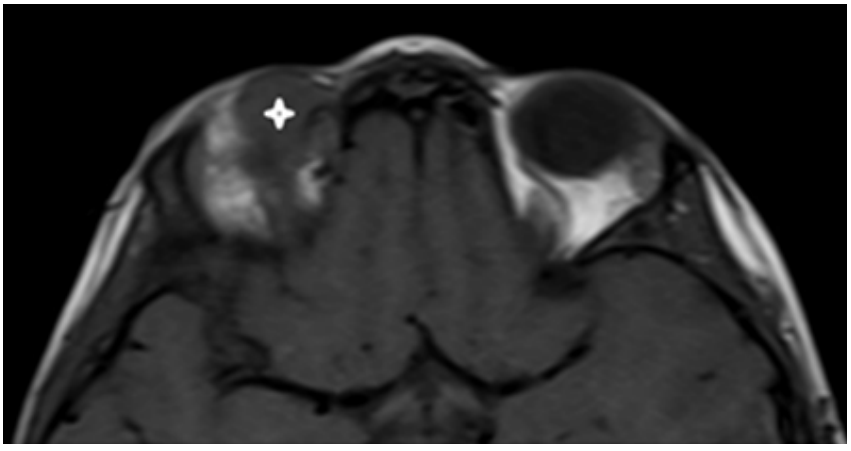

Figure $2 \mathrm{a}$ TI axial MRI of the mass.

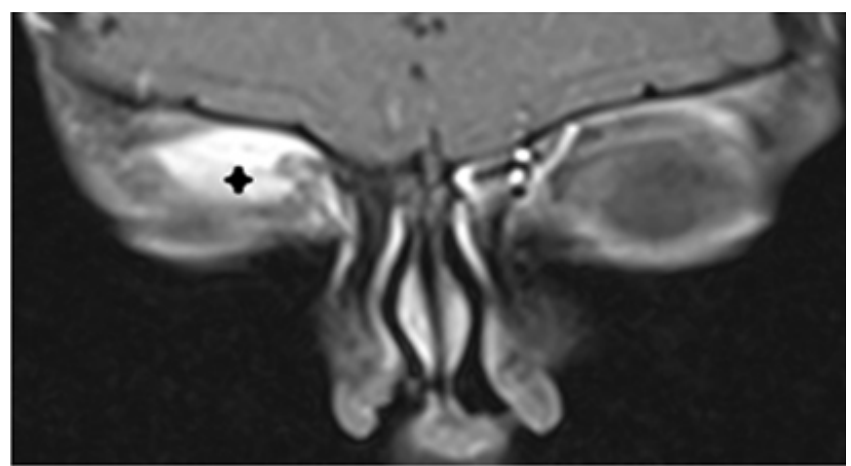

Figure $\mathbf{2 b} \mathrm{TI}$ coronar MRI with contrast of the mass.

Orbital MRI revealed a well-circumscribed mass lesion which is located extraconal, superior part of right orbit and behind the medial canthus, isointense with respect to brain parenchym on T1 and T2A, showed diffuse homogeneous contrast enhancement after intravenous injection of contrast. Lesion's approximate size was $22 \times 16 \times 10 \mathrm{~mm}$ and pushing the eyeball and superior rectus muscle inferiorly. The mass caused to erosion in frontal bone. WBC: $17.690 / \mathrm{dL}, \mathrm{RBC}$ : RBC: $1.55 \mathrm{~K} / \mathrm{dL}, \mathrm{Hgb}: 4.6 \mathrm{~g} / \mathrm{dL}, \mathrm{Htc}: 14 \mathrm{~g} / \mathrm{L}, \mathrm{Plt}: 49 \mathrm{~K} / \mathrm{dL}$ were in complete blood account. Lactate dehydrogenase was $831 \mathrm{U} / \mathrm{L}$ in biochemical examination. Peripheral blood showed an increased total leucocyte count $\left(17,000 \mathrm{cells} / \mathrm{mm}^{3}\right)$. Her differential leucocyte count revealed $5 \%$ neutrophils, $12 \%$ lymphocytes, $2 \%$ eosinophils, $2 \%$ monocytes, $79 \%$ myeloblasts and immature myeloid precursors which included promyelocytes, myelocytes and metamyelocytes. Patient was $t$ $(8 ; 21)$ positive and the subtype was AML M2. The bone marrow aspirate showed hyper cellular marrow smears with replacement of haemopoietic cells by blasts and blasts were Sudan Black B positive. Patient referred to paediatric haematology clinic and treated with standart cytarabine and anthracycline based chemotherapy protocole and re-evaluated at the end of the 2nd week of the treatment. Blasts were decreased to $15 \%$ and proptosis disappeared in examination. And the blasts decreased below to 5\% at the end of 4 th week and the choloma disappeared in the orbital MRI.

\section{Discussion}

Granulocytic sarcomas are associated with AML (FAB sub Types M2, M4 and M5); they may also be associated with myelodysplastic syndrome (MDS) and myeloproliferative disorders (MPS). The WHO has classified granulocytic sarcomas into 3 main types, depending on the degree of maturation (i) Blastic- which is composed mainly of myeloblasts (ii) Immature-which is composed of myeloblasts and promyelocytes and (iii) Differentiated- which is composed of promyelocytes and more mature myeloid cells.7-9 Granulocytic sarcoma develops preferentially in bones or periosteum (especially in the skull) and it may lead to proptosis. The proptosis in these cases is mainly caused by leukaemic infiltrates, a retro bulbar haemorrhage, an orbital muscle infiltration or a venous blockage. Other presentations which are caused by an orbital involvement include ptosis, lacrimal gland involvement, conjunctival masses and an iridic and a diffuse uveal involvement. Most of the reported cases had decreased visual acuity and restricted extra-ocular movements. ${ }^{7,10}$

Patients with isolated GS have good prognosis if they are treated with current AML therapy. For those patients who have GS in addition to a marrow involvement, the patients with disease which is limited to the skin do worse than those without GS; those with AML that involves sites other than skin (e.g., orbit, head, and neck), have similar prognosis as those of patients who have bone marrow leukaemia alone. ${ }^{11}$ However, in a study that reported from Turkey, Gozdasoglu et al. ${ }^{6}$ analyzed 33 paediatric patients and found that this group GS with AML behaves poorly compared to AML patients without GS. An extra-medullary leukaemic infiltration is a bad prognostic sign, because it might act as a reservoir for proliferation of leukaemic cells and an eventual relapse. In such cases, fine needle aspiration cytology may be a useful diagnostic tool for making an early diagnosis. Cytological preparations give us better morphological details of blasts; the identification of Auer rods and cytochemistry can help in clinching the diagnosis of an undetected AML. ${ }^{12}$ In our case, general blood pictures, bone marrow morphologies and special staining (Sudan Black B) and radiologic evaluations helped in making the diagnosis. And standart chemotherapy reveal remission in both bone morrow and the sarcomatoid ophthalmic mass within 2 week; however the presence of the chloroma defined as poor prognostic sign in previous reports.

\section{Conclusion}

A variety of ophthalmologic findings can be seen in leukemia's. These findings can be observed before establishment of diagnosis or patients on remission. Therefore, it is essential that all physicians should know and consider while evaluating leukemic patients. Careful ophthalmologic examination plays crucial role on the timely diagnosis of many curable disorders. Thus, permanent visual loss and functional ophthalmologic disorders of leukemic patients with high survival rate can be prevented. That is because detailed and careful ophthalmologic examination has vital importance on the management of all leukemic patients.

\section{Acknowledgments}

None.

\section{Funding}

None.

\section{Conflicts of interest}

The authors declare there is no conflict of interests.

\section{References}

1. Murthy R, Vemuganti GK, Honavar SG, et al. Extramedullary leukemia in children presenting with proptosis. J Hematol Oncol. 2009;2:4.

2. Stein-Wexler R, Wootton-Gorges SL, West DC. Orbital granulocytic sarcoma: an unusual presentation of acute myelocytic leukemia. Pediatr Radiol. 2003;33(2):136-139. 
3. Stockl FA, Dolmetsch AM, Saornil MA, et al. Orbital granulocytic sarcoma. Br J Ophthalmol. 1997;81(12):1084-1088.

4. Uyesugi WY, Watabe J, Petermann G. Orbital and facial granulocytic sarcoma (chloroma): a case report. Pediatr Radiol. 2000;30:276-278.

5. Rappaport H. Atlas of Tumor Pathology. Washington DC: Armed Forces Institute of Pathology, Tumors of the hematopoeitic system; 1966:241243.

6. Gozdasoglu S, Yavuz G, Unal E, et al. Orbital granulocytic sarcoma and AML with poor prognosis in Turkish children. Leukemia. 2002;16(5):962.

7. Thakur B, Varma K, Misra V, et al. Granulocytic sarcoma presenting as an orbital mass: report of two cases. J Clin Diagn Res. 2013;7(8):1704-1706.

8. Jaffe ES, Harris NL, Stein H. World health organisation classification of tumours. Tumours of haematopoietic and lymphoid tissues 105; 2001.
9. Kundu S, Chatterjee S, Mondol D, et al. Extramedullary granulocytic sarcoma. Indian J Med Paediatr Oncol. 2008;29:28-30.

10. Bangerter M, Hildebrand A, Waidmann O, et al. Diagnosis of Granulocytic Sarcoma by Fine-Needle Aspiration Cytology. Acta Haematol. 2000;103:102-108.

11. Dusenbery KE, Howells WB, Arthur DC, et al. Extramedullary leukaemia in children with newly diagnosed acute myeloid leukemia: a report from the Children's Cancer Group. J Pediatr Hematol Oncol. 2003;25(10):760 768.

12. Tangri R, Prakash N, Khurana A. Myeloid sarcoma of the orbit. J Cytol. 2008;25:111-112. 